\title{
Synthesis of sporiolide B from D-glucal
}

\author{
Qi Chen and Yuguo $\mathrm{Du}^{*}$ \\ State Key Laboratory of Environmental Chemistry and Ecotoxicology, Research Center for Eco-Environmental Sciences, \\ Chinese Academy of Sciences, Beijing 100085, PR China
}

Received 4 March 2007; received in revised form 19 April 2007; accepted 20 April 2007

Available online 29 April 2007

\begin{abstract}
A total synthesis of the 12-membered ring natural macrolide, sporiolide B, was achieved from D-glucal in 17 steps with $4.8 \%$ overall yield. The required stereochemical configuration at C-3 and C-5 in sporiolide B was easily introduced by applying a Mitsunobu reaction on the chiral template D-glucal. Yamaguchi esterification and ring closing metathesis greatly improved the access to the target compound.

(C) 2007 Elsevier Ltd. All rights reserved.
\end{abstract}

Keywords: Total synthesis; Macrolides; Sporiolide B; D-Glucal; Natural products

\section{Introduction}

Marine microorganisms, such as bacteria, fungi, and microalgae, which have proved to be a rich source of structurally novel and biologically active secondary metabolites, are attracting increasing attention as a potential source of new pharmaceuticals and pharmaceutical leads. ${ }^{1}$ In 2004, from the culture broth of a fungus Cladosporium sp. separated from an Okinawan marine brown alga Actinotrichia fragilis, Kobayashi reported the isolation of a 12-membered ring macrolide sporiolide B (1) and proposed that structurally it corresponds to the 3-O-methyl ether of pandangolide $1(2)^{2}$ based on spectroscopic analyses (Chart 1). ${ }^{3}$

Sporiolide B exhibits cytotoxicity against L1210 cells $\left(\mathrm{IC}_{50}=0.81 \mu \mathrm{g} / \mathrm{mL}\right)$ and antibacterial activity against Micrococcus luteus $(\mathrm{MIC}=16.7 \mu \mathrm{g} / \mathrm{mL})$. Attracted by its various biological activities, we already completed the first total synthesis of sporiolide B from D-xylose in $3.5 \%$ overall yield. ${ }^{4}$ In our ongoing project, we needed to synthesize larger quantities of sporiolide B for bioactivity screening. However, in large scale preparations with our previous procedure, we realized that a number

\footnotetext{
*Corresponding author. Tel.: +86 10 62849126; fax: +86 10 62923563; e-mail: duyuguo@rcees.ac.cn
}

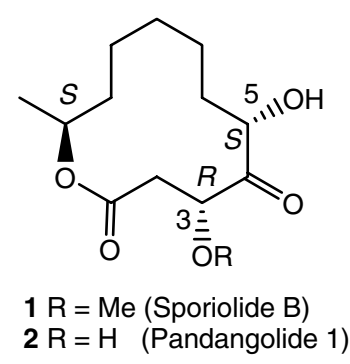

Chart 1. Structures of sporiolide B (1) and pandangolide 1 (2).

of toxic reagents $\left(\mathrm{HgAc}_{2}\right.$ and $\left.\mathrm{KCN}\right)$ were used. We thus decided to explore an alternative strategy toward the target molecule. Herein, a facile total synthesis of sporiolide B from 1,5-anhydro-2-deoxy-D-arabino-hex-1enitol (D-glucal) as starting material is now reported.

\section{Results and discussion}

As outlined in Chart 2, sporiolide B (1) could be prepared from the lactone precursor $\mathbf{3}$ available from intramolecular ring closure metathesis of diene $\mathbf{4}$ after Yamaguchi esterification of alcohol $\mathbf{5}$ and acid $\mathbf{6}$. Key intermediate 6 could be derived from the well functionalized lactone 7 , which would be prepared from commercially available D-glucal. 


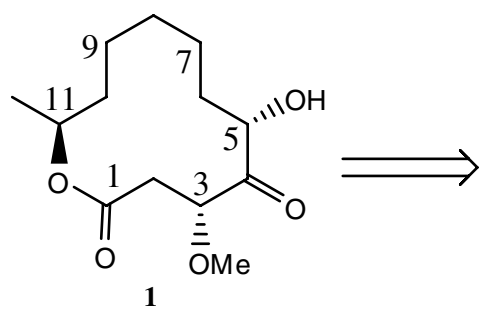

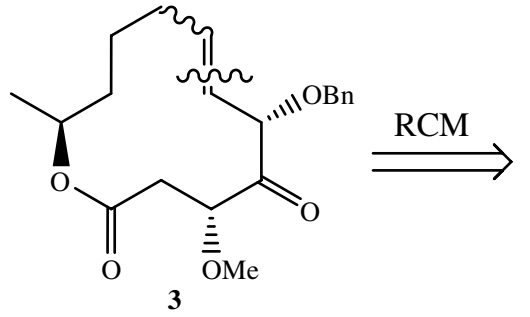<smiles>C=CCCC[C@H](C)OC(=O)C[C@H](OC)C(=O)[C@H](O)C=C</smiles>

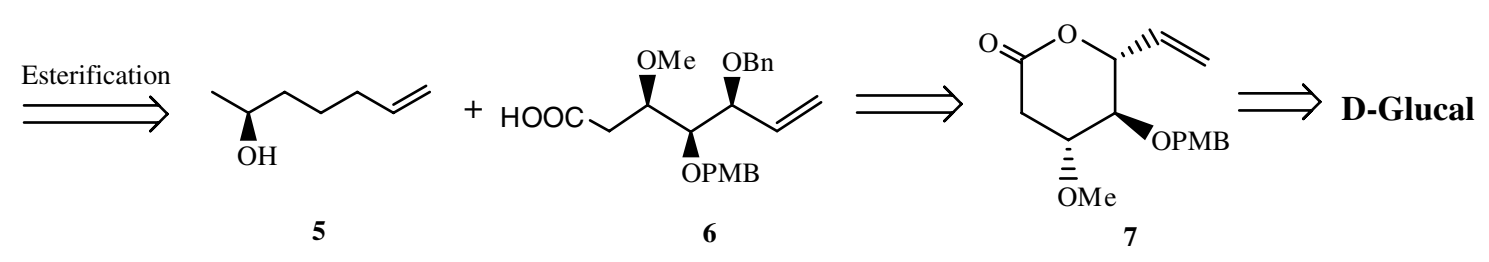

Chart 2. Retrosynthetic analysis of sporiolide B (1).

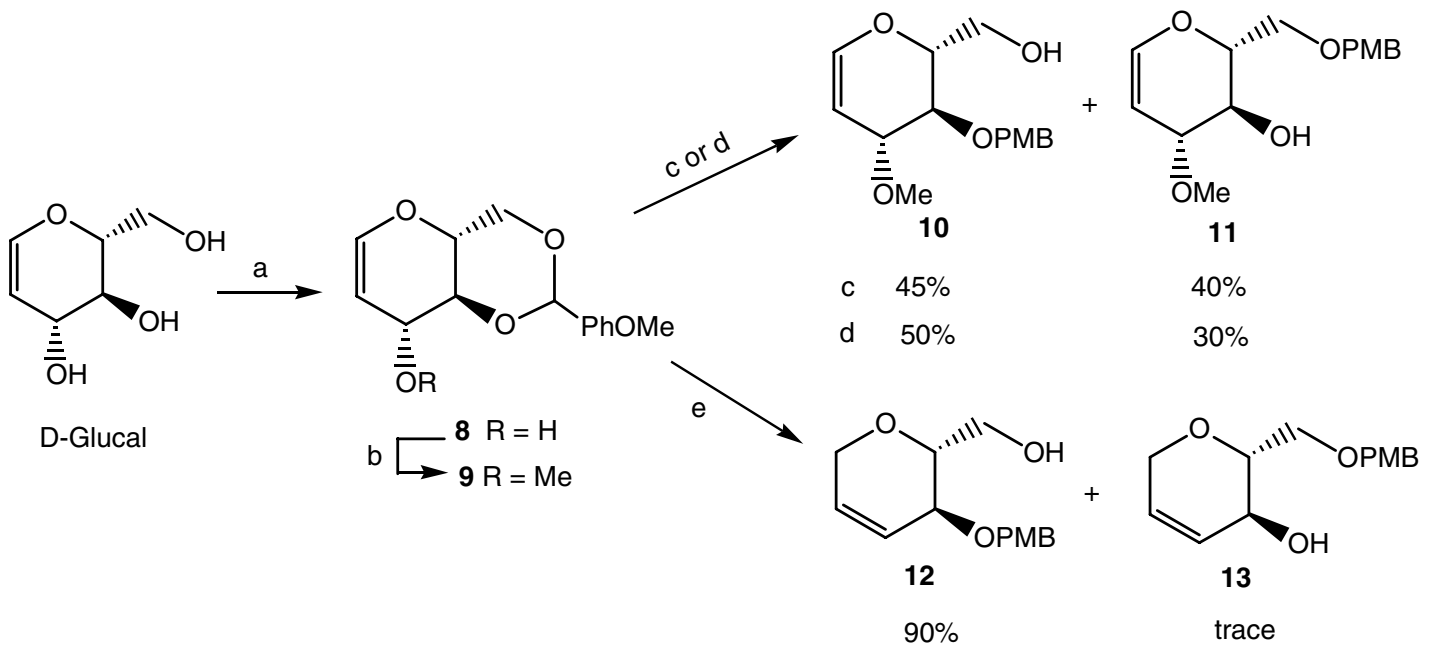

Scheme 1. Attempted cleavage of $p$-anisaldehyde acetal by regioselective reduction. Reagents and conditions: (a) Ref. 5; (b) MeI, NaH, DMF, 2 h; $95 \%$; (c) DIBAL-H, DCM, $-78{ }^{\circ} \mathrm{C}$ to rt, $2 \mathrm{~h}, 45 \%$ for 10, $40 \%$ for 11; (d) $\mathrm{LiAlH}_{4}, \mathrm{AlCl}_{3}, \mathrm{THF}, 75{ }^{\circ} \mathrm{C}, 6 \mathrm{~h}, 50 \%$ for 10, 30\% for 11; (e) $\mathrm{NaCNBH}$, TMSCl, MeCN, 4 h, $90 \%$ for 12 .

Initially, we tried to prepare $\mathbf{1 0}$ from $\mathbf{9}$, which was obtained by methylation of the known D-glucal derivative $\mathbf{8}^{5}$ through regioselective reductive cleavage of $p$-anisaldehyde acetal as illustrated in Scheme 1. Unfortunately, a poor selectivity was obtained using either DIBAL- $\mathrm{H}^{5,6}$ or $\mathrm{LiAlH}_{4}-\mathrm{AlCl}_{3}{ }^{7}$ as reducing agents. When 9 was treated with $\mathrm{NaCNBH}_{3}-\mathrm{TMSCl}_{1}^{8}{ }^{12}$ involving double bond migration was obtained unexpectedly in an excellent yield $(90 \%)$, and its structure was confirmed unambiguously by ${ }^{1} \mathrm{H}$ NMR spectroscopy.

To solve this problem, we turned our attention to another synthetic route as shown in Scheme 2. Silylated 14 was easily obtained from D-glucal in five steps according to our previous report. ${ }^{9}$ Thus, desilylation with TBAF gave alcohol 15, which was methylated with $\mathrm{MeI}$ and $\mathrm{NaH}$ resulting in the glucal derivative 16. Compound 16 was subjected to PCC oxidation ${ }^{10}$ at $45^{\circ} \mathrm{C}$ in the presence of silica gel to give the key lactone 7 in good yield (67\% in three steps). Treatment of 7 with $\mathrm{NaOMe}$ in $\mathrm{MeOH}$ gave the methyl ester 17, in which the free $(R)$ $\mathrm{OH}$ was inverted into the precursor $(S)$-benzoate under Mitsunobu reaction conditions. ${ }^{11}$ Debenzoylation of this precursor with $\mathrm{NaOMe}$ in methanol $(\rightarrow \mathbf{1 8})$, followed by benzylation with benzyl 2,2,2-trichloroacetimidate $(\rightarrow \mathbf{1 9}),{ }^{12}$ and hydrolysis with $\mathrm{LiOH}$, furnished acid 6. Esterification of $\mathbf{6}$ with $(S)$-6-hepten-2-ol $(\mathbf{5})^{13}$ under Yamaguchi's conditions ${ }^{14}$ afforded the diene derivative 20 (78\% from 19), which was subjected to PMB cleavage with DDQ ${ }^{15}$ and Dess-Martin periodinane ${ }^{16}$ oxidation, to give the key intermediate $\mathbf{4}$ in $77 \%$ yield and two steps. Diene 4 was then exposed to the Grubbs catalyst $\left[\mathrm{PHCH}=\mathrm{RuCl}\left(\mathrm{PCy}_{3}\right)_{2}, \quad 30 \mathrm{~mol} \%\right]^{17}$ undergoing an intramolecular ring closure metathesis (RCM) to produce macrolactone $\mathbf{3}$ as a $E, Z$ mixture $(E / Z=2: 1)$. Final hydrogenation of 3 with $\mathrm{H}_{2}$ in the presence of $\mathrm{Pd} / \mathrm{C}$ simultaneously cleaved the benzyl ether and 


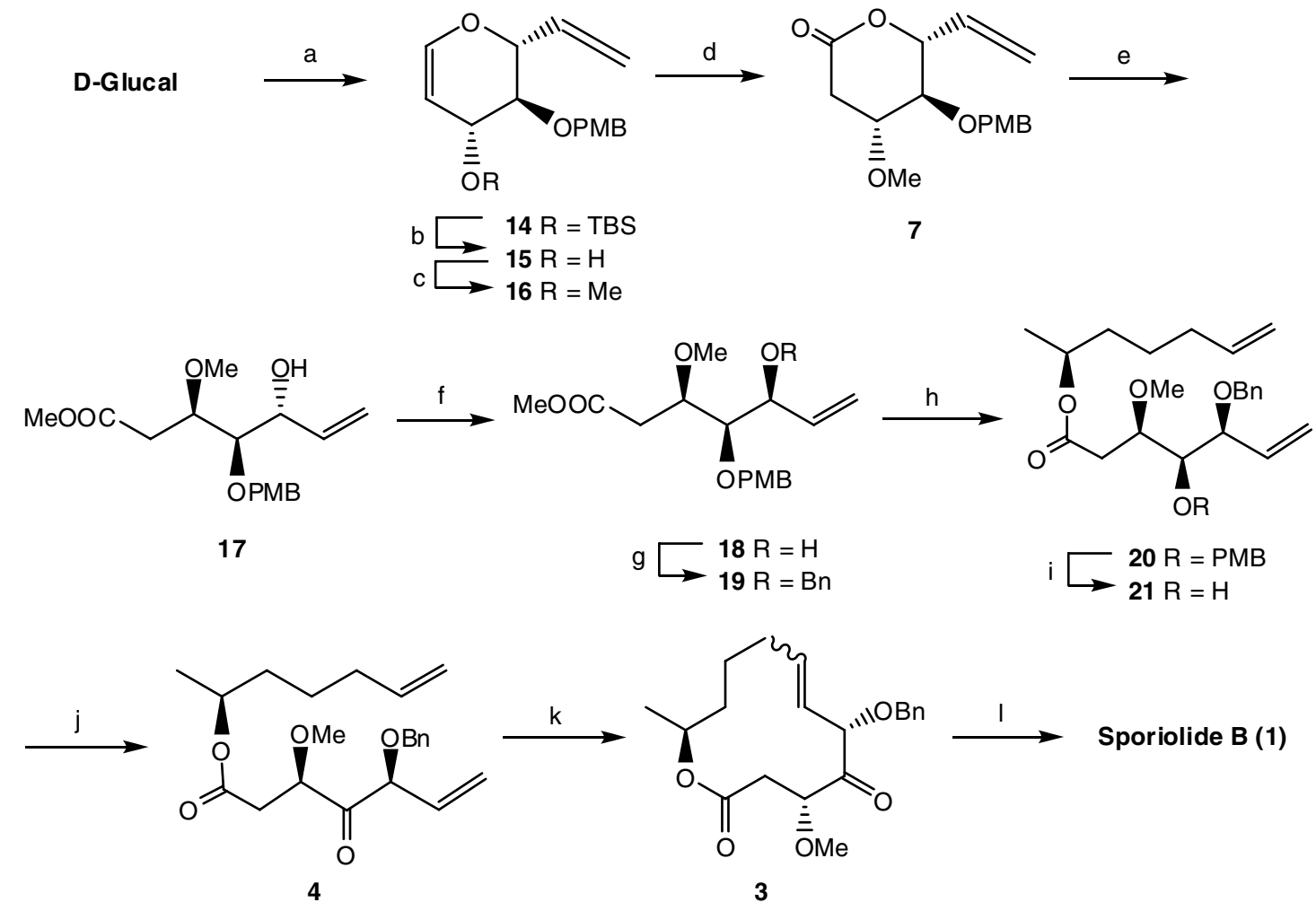

Scheme 2. Reagents and conditions: (a) Ref. 10, 44\%; (b) TBAF, THF, 2 h, 95\%; (c) MeI, NaH, DMF, 2 h, 95\%; (d) PCC, silica gel, DCM, $45{ }^{\circ} \mathrm{C}$, 6 h, 70\%; (e) NaOMe, MeOH, 3 h, 83\%; (f) (i) $\mathrm{Ph}_{3} \mathrm{P}$, DEAD, PhCOOH, THF, 2 h; (ii) NaOMe, MeOH, 3 h, $81 \%$ in two steps; (g) BnOC(NH)CCl, TMSOTf, DCM, 3 h, 75\%; (h) (i) LiOH, THF/water, 12 h; (ii) (s)-6-hepten-2-ol, 2,4,6-trichlorobenzoyl chloride, TEA, DMAP, THF, 18 h, $78 \%$ in two steps; (i) DDQ, DCM/water, 2 h, 93\%; (j) Dess-Martin periodinane, DCM, 3 h, 83\%; (k) 30\% $\mathrm{PhCH}=\mathrm{RuCl}_{2}\left(\mathrm{PCy}_{3}\right)_{2}, \mathrm{DCM}$, refluxing, $24 \mathrm{~h}, 70 \%$ $(E / Z=2: 1) ;(1) \mathrm{H}_{2}, \mathrm{Pd} / \mathrm{C}, \mathrm{MeOH}, 12 \mathrm{~h}, 81 \%$.

saturated the double bond yielding sporiolide B, identical in every respect to the natural product. ${ }^{3}$

The effects of sporiolide B against human MDA231, BEL-7402, and Hela cell growth were investigated with different methods. ${ }^{3,18}$ Unfortunately, under these testing conditions, sporiolide B did not show significant inhibition on proliferation of either human MDA231, BEL7402 , or Hela cell lines at $0.25-1.0 \mu \mathrm{g} / \mathrm{mL}$ for 24,48 , and $72 \mathrm{~h}$, respectively.

In summary, we have achieved the total synthesis of sporiolide B in 17 steps and $4.8 \%$ overall yield. The required stereochemical configuration at $\mathrm{C}-3$ and $\mathrm{C}-5$ in sporiolide B was successfully controlled by using a Mitsunobu reaction to invert the stereochemistry at C-5 of a glucal-derived compound. Yamaguchi esterification and ring closing metathesis greatly improved the target synthesis.

\section{Experimental}

\subsection{General methods}

Optical rotations were determined at $25^{\circ} \mathrm{C}$ with a Perkin-Elmer Model 241-Mc automatic polarimeter, and $[\alpha]_{\mathrm{D}^{-v a l u e s}}$ are in units of $10^{-1} \mathrm{deg} \mathrm{cm}^{2} \mathrm{~g}^{-1} \cdot{ }^{1} \mathrm{H}$ NMR, ${ }^{13} \mathrm{C}$ NMR, and ${ }^{1} \mathrm{H}-{ }^{1} \mathrm{H},{ }^{1} \mathrm{H}-{ }^{13} \mathrm{C}$ COSY spectra were recorded with a Bruker ARX 400 spectrometer for solns in $\mathrm{CDCl}_{3}$. Chemical shifts are given in parts per million downfield from internal $\mathrm{Me}_{4} \mathrm{Si}$. Mass spectra were measured using a MALDI TOF mass spectrometer with $\alpha$-cyano-4-hydroxycinnamic acid (CCA) as matrix, or recorded with a VG PLATFORM mass spectrometer using the $\operatorname{ESI}(-)$ technique to introduce the sample. TLC was performed on silica gel $\mathrm{HF}_{254}$ with detection by charring with $30 \% \mathrm{H}_{2} \mathrm{SO}_{4}$ in $\mathrm{MeOH}$ or in some cases by UV detection. Column chromatography was conducted by elution of a column of silica gel (100-200 mesh) with EtOAc-petroleum ether $\left(60-90^{\circ} \mathrm{C}\right)$. Solns were concentrated at $<60{ }^{\circ} \mathrm{C}$ under diminished pressure.

\section{2. (2R,3S)-2-Hydroxymethyl-3-(4-methoxybenzyloxy)- 3,6-dihydro-2H-pyran (12)}

To a stirred soln of $\mathbf{8}^{5}(2.2 \mathrm{~g}, 8.4 \mathrm{mmol})$ in anhyd DMF $(15 \mathrm{~mL})$ was added $\mathrm{NaH}(400 \mathrm{mg}, 16.4 \mathrm{mmol})$ at $0{ }^{\circ} \mathrm{C}$, 30 min later, methyl iodide $(0.6 \mathrm{~mL}, 9 \mathrm{mmol})$ was added dropwise under the same reaction conditions. After stirring at $\mathrm{rt}$ for $2 \mathrm{~h}$, the reaction mixture was poured into ice water $(30 \mathrm{~mL})$ and extracted with EtOAc 
$(2 \times 50 \mathrm{~mL})$. The combined organic layers were dried over anhyd $\mathrm{Na}_{2} \mathrm{SO}_{4}$ and concentrated under diminished pressure. Purification of the residue on column chromatography (7:1 petroleum ether-EtOAc) afforded $\mathbf{9}(2.2 \mathrm{~g}$, $95 \%)$ as a solid. To a stirred soln of $9(0.5 \mathrm{~g}, 1.8 \mathrm{mmol})$ in anhyd $\mathrm{MeCN}(10 \mathrm{~mL})$ at $0{ }^{\circ} \mathrm{C}$ was added $\mathrm{TMSCl}$ $(1.5 \mathrm{~mL}, 10.8 \mathrm{mmol})$ portionwise, and then $\mathrm{NaCNBH}_{3}$ (754 mg, $10.8 \mathrm{mmol}$ ) was added under the same conditions. The resulting mixture was diluted with $\mathrm{CH}_{2} \mathrm{Cl}_{2}$ $(50 \mathrm{~mL}) 4 \mathrm{~h}$ later and washed with satd aq $\mathrm{NaHCO}_{3}$ and aq $\mathrm{NaCl}$. After drying over anhyd $\mathrm{Na}_{2} \mathrm{SO}_{4}$ and concentration under diminished pressure, the residue was purified by column chromatography (3:1 petroleum ether-EtOAc) to give $\mathbf{1 2}(400 \mathrm{mg}, 90 \%)$ as a colorless syrup: $[\alpha]_{\mathrm{D}}^{25}-10\left(c 1, \mathrm{CHCl}_{3}\right) ;{ }^{1} \mathrm{H}$ NMR $\left(\mathrm{CDCl}_{3}\right): \delta$ $7.26(\mathrm{~d}, 2 \mathrm{H}, J 8.5 \mathrm{~Hz}, \mathrm{Ph}), 6.88(\mathrm{~d}, 2 \mathrm{H}, J 8.6 \mathrm{~Hz}, \mathrm{Ph})$, $5.93(\mathrm{~d}, 1 \mathrm{H}, J 10.4 \mathrm{~Hz}, \mathrm{H}-3), 5.85(\mathrm{~d}, 1 \mathrm{H}, J 10.5 \mathrm{~Hz}$, $\mathrm{H}-2), 4.60,4.47$ (2d, 2H, $\left.J 11.2 \mathrm{~Hz}, \mathrm{PhCH}_{2}\right), 4.24-4.15$ $(\mathrm{m}, 2 \mathrm{H}, \mathrm{H}-1 \mathrm{a}$ and $\mathrm{H}-1 \mathrm{~b}), 3.99(\mathrm{~d}, 1 \mathrm{H}, J 8.7 \mathrm{~Hz}, \mathrm{H}-4)$, $3.86(\mathrm{dd}, 1 \mathrm{H}, J 2.9,11.6 \mathrm{~Hz}, \mathrm{H}-6 \mathrm{~b}), 3.81(\mathrm{~s}, 3 \mathrm{H}$, $\left.\mathrm{OCH}_{3}\right), 3.68(\mathrm{dd}, 1 \mathrm{H}, J$ 5.6, $11.6 \mathrm{~Hz}, \mathrm{H}-6 \mathrm{a}), 3.49$ 3.45 (m, 1H, H-5), 1.86 (br s, $1 \mathrm{H}, \mathrm{OH}$ ). Anal. Calcd for $\mathrm{C}_{14} \mathrm{H}_{18} \mathrm{O}_{4}$ : $\mathrm{C}, 67.18 ; \mathrm{H}, 7.25$. Found: $\mathrm{C}, 67.33 ; \mathrm{H}$, 7.31.

\section{3. (2R,3S,4R)-3-(4-Methoxybenzyloxy)-2-vinyl-3,4- dihydro-2H-pyran-4-ol (15)}

To a soln of silyl ether $14^{9}(4.5 \mathrm{~g}, 12 \mathrm{mmol})$ in THF $(50 \mathrm{~mL})$ was added TBAF $(24 \mathrm{~mL}$ of a $1 \mathrm{M}$ soln in THF, $24 \mathrm{mmol}$ ) at $0{ }^{\circ} \mathrm{C}$. The mixture was stirred under these conditions for $30 \mathrm{~min}$, followed by additional $2 \mathrm{~h}$ stirring at rt, at the end of which time, TLC indicated completion of the reaction. Then the reaction mixture was concentrated under diminished pressure and the residue was subjected to column chromatography (4:1 petroleum ether-EtOAc) to furnish $15(3.0 \mathrm{~g}, 95 \%)$ as a colorless syrup: $[\alpha]_{\mathrm{D}}^{25}-17\left(c 1, \mathrm{CHCl}_{3}\right) ;{ }^{1} \mathrm{H} \mathrm{NMR}$ $\left(\mathrm{CDCl}_{3}\right): \delta 7.28(\mathrm{~d}, 2 \mathrm{H}, J 8.1 \mathrm{~Hz}, \mathrm{Ph}), 6.89(\mathrm{~d}, 2 \mathrm{H}, J$ $7.7 \mathrm{~Hz}, \mathrm{Ph}), 6.39(\mathrm{~d}, 1 \mathrm{H}, J 6.0 \mathrm{~Hz}, \mathrm{OCH}=\mathrm{CH}), 6.05-$ $5.92\left(\mathrm{~m}, 1 \mathrm{H}, \mathrm{C} H=\mathrm{CH}_{2}\right), 5.47(\mathrm{~d}, 1 \mathrm{H}, J 17.3 \mathrm{~Hz}$, $\left.\mathrm{CH}=\mathrm{CH}_{\mathrm{a}} \mathrm{H}_{\mathrm{b}}\right), 5.34\left(\mathrm{~d}, 1 \mathrm{H}, J 10.6 \mathrm{~Hz}, \mathrm{CH}=\mathrm{CH}_{\mathrm{a}} H_{\mathrm{b}}\right)$, $4.78(\mathrm{dd}, 1 \mathrm{H}, J 2.6,6.0 \mathrm{~Hz}, \mathrm{OCH}=\mathrm{CH}), 4.74(\mathrm{~d}, 1 \mathrm{H}, J$ $11.3 \mathrm{~Hz}, \mathrm{PhCH}), 4.61(\mathrm{~d}, 1 \mathrm{H}, J 11.2 \mathrm{~Hz}, \mathrm{PhCH}), 4.35$ $(\mathrm{t}, 1 \mathrm{H}, J 7.2 \mathrm{~Hz}, \mathrm{CHOPMB}), 4.28(\mathrm{~s}, 1 \mathrm{H}, \mathrm{OCHCH}=$ $\left.\mathrm{CH}_{2}\right), 3.80\left(\mathrm{~s}, 3 \mathrm{H}, \mathrm{OCH}_{3}\right), 3.43(\mathrm{t}, 1 \mathrm{H}, J 6.9 \mathrm{~Hz}$, $\mathrm{C} H \mathrm{OH}) ;{ }^{13} \mathrm{C}$ NMR $\left(100 \mathrm{MHz}, \mathrm{CDCl}_{3}\right): \delta 159.9,144.6$, 134.5, 130.5, 130.0 (2C), 118.6, 114.4 (2C), 103.2, 80.5, 78.1, 73.8, 68.7, $55.7\left(\mathrm{OCH}_{3}\right)$. Anal. Calcd for $\mathrm{C}_{15} \mathrm{H}_{18} \mathrm{O}_{4}$ : C, 68.68; H, 6.92. Found: C, 69.09; H, 6.84.

\section{4. $(2 R, 3 S, 4 R)-4-M e t h o x y-3-(p-m e t h o x y b e n z y l o x y)-2-$ vinyl-3,4-dihydro-2H-pyran (16)}

To a stirred soln of $\mathbf{1 5}(1.1 \mathrm{~g}, 4.2 \mathrm{mmol})$ in anhyd DMF $(15 \mathrm{~mL})$ was added $\mathrm{NaH}(200 \mathrm{mg}, 8.2 \mathrm{mmol})$ portion- wise at $0{ }^{\circ} \mathrm{C}$ and 30 min later, methyl iodide $(0.3 \mathrm{~mL}$, $4.5 \mathrm{mmol}$ ) was added dropwise under the same conditions. After stirring at $\mathrm{rt}$ for $2 \mathrm{~h}$, the reaction mixture was poured into ice water $(20 \mathrm{~mL})$ and extracted with EtOAc $(2 \times 40 \mathrm{~mL})$. The combined organic layer was dried over anhyd $\mathrm{Na}_{2} \mathrm{SO}_{4}$ and concentrated under diminished pressure. Purification of the residue by column chromatography (7:1 petroleum ether-EtOAc) afforded $16(1.1 \mathrm{~g}, 95 \%)$ as a solid: $[\alpha]_{\mathrm{D}}^{25}-18(c 0.5$, $\left.\mathrm{CHCl}_{3}\right) ;{ }^{1} \mathrm{H}$ NMR $\left(\mathrm{CDCl}_{3}\right): \delta 7.27(\mathrm{~d}, 2 \mathrm{H}, J 8.4 \mathrm{~Hz}$, $\mathrm{Ph}), 6.87(\mathrm{~d}, 2 \mathrm{H}, J 8.3 \mathrm{~Hz}, \mathrm{Ph}), 6.41(\mathrm{~d}, 1 \mathrm{H}, J 6.1 \mathrm{~Hz}$, $\mathrm{OCH}=\mathrm{C}), 6.07-5.93\left(\mathrm{~m}, 1 \mathrm{H}, \mathrm{CH}=\mathrm{CH}_{2}\right), 5.40(\mathrm{~d}, 1 \mathrm{H}$, $\left.J \quad 17.2 \mathrm{~Hz}, \mathrm{CH}=\mathrm{CH}_{\mathrm{a}} \mathrm{H}_{\mathrm{b}}\right), 5.29(\mathrm{~d}, 1 \mathrm{H}, J 10.5 \mathrm{~Hz}$, $\left.\mathrm{CH}=\mathrm{CH}_{\mathrm{a}} H_{\mathrm{b}}\right), 4.86(\mathrm{dd}, 1 \mathrm{H}, J 2.5,6.1 \mathrm{~Hz}, \mathrm{OCH}=\mathrm{CH})$, $4.71(\mathrm{~d}, 1 \mathrm{H}, J 11.0 \mathrm{~Hz}, \mathrm{PhCH}), 4.62(\mathrm{~d}, 1 \mathrm{H}, J 11.0 \mathrm{~Hz}$, $\mathrm{PhCH}$ ), 4.29 (t, $1 \mathrm{H}, J 7.5 \mathrm{~Hz}, \mathrm{CHOPMB}), 3.96(\mathrm{~d}, 1 \mathrm{H}$, $\left.J 6.0 \mathrm{~Hz}, \mathrm{OCHCH}=\mathrm{CH}_{2}\right), 3.80\left(\mathrm{~s}, 3 \mathrm{H}, \mathrm{OCH}_{3}\right.$ in PMB), 3.49 (dd, 1H, J 6.7, 8.5 Hz, CHOMe), 3.39 (s, $\left.3 \mathrm{H}, \mathrm{OCH}_{3}\right) ;{ }^{13} \mathrm{C}$ NMR $\left(100 \mathrm{MHz}, \mathrm{CDCl}_{3}\right): \delta 159.7$, 143.7, 133.7, 129.6, 128.9 (2C), 118.6, 113.1 (2C), 99.2, 98.8, 77.1, 76.6, 72.4, $55.8\left(\mathrm{PhOCH}_{3}\right), 54.5\left(\mathrm{OCH}_{3}\right)$. Anal. Calcd for $\mathrm{C}_{16} \mathrm{H}_{20} \mathrm{O}_{4}$ : C, 69.54; H, 7.30. Found: C, 69.81; H, 7.22.

\section{5. $(4 R, 5 S, 6 R)-4-M e t h o x y-5-(p$-methoxybenzyloxy)-6- vinyl-tetrahydropyran-2-one (7)}

To a soln of $\mathbf{1 6}(880 \mathrm{mg}, 3.2 \mathrm{mmol})$ in $\mathrm{CH}_{2} \mathrm{Cl}_{2}(100 \mathrm{~mL})$ was added PCC $(2.1 \mathrm{~g}, 9.6 \mathrm{mmol})$ and silica gel $(2.6 \mathrm{~g})$. The stirred suspension was refluxed for $6 \mathrm{~h}$, then cooled and filtered through Celite. The Celite pad was washed several times with EtOAc and the combined filtrates were concentrated. The crude product was purified by column chromatography (4:1 petroleum ether-EtOAc) to give $7(650 \mathrm{mg} ; 70 \%)$ as a colorless syrup: $[\alpha]_{\mathrm{D}}^{25}-15$ (c $\left.0.8, \mathrm{CHCl}_{3}\right) ;{ }^{1} \mathrm{H}$ NMR $\left(\mathrm{CDCl}_{3}\right): \delta 7.25(\mathrm{~d}, 2 \mathrm{H}, J$ $8.2 \mathrm{~Hz}, \mathrm{Ph}), 6.90(\mathrm{~d}, 2 \mathrm{H}, J 8.2 \mathrm{~Hz}, \mathrm{Ph}), 5.98-5.82(\mathrm{~m}$, $\left.1 \mathrm{H}, \mathrm{CH}=\mathrm{CH}_{2}\right), 5.43\left(\mathrm{~d}, 1 \mathrm{H}, J 17.2 \mathrm{~Hz}, \mathrm{CH}=\mathrm{CH}_{\mathrm{a}} \mathrm{H}_{\mathrm{b}}\right)$, $5.30\left(\mathrm{~d}, 1 \mathrm{H}, J 10.6 \mathrm{~Hz}, \mathrm{CH}=\mathrm{CH}_{\mathrm{a}} H_{\mathrm{b}}\right), 4.62(\mathrm{~d}, 1 \mathrm{H}, J$ $11.0 \mathrm{~Hz}, \mathrm{PhCH}), 4.61-4.57$ (m, 1H, CHOPMB), 4.55 $(\mathrm{d}, 1 \mathrm{H}, J 11.0 \mathrm{~Hz}, \mathrm{PhCH}), 3.81\left(\mathrm{~s}, 3 \mathrm{H}, \mathrm{PhOCH}_{3}\right)$, $3.76\left(\mathrm{dd}, 1 \mathrm{H}, J 3.6,7.8 \mathrm{~Hz}, \mathrm{OC} H \mathrm{CH}=\mathrm{CH}_{2}\right), 3.54(\mathrm{dd}$, $1 \mathrm{H}, J 3.4,7.5 \mathrm{~Hz}, \mathrm{CHOMe}), 3.36\left(\mathrm{~s}, 3 \mathrm{H}, \mathrm{OCH}_{3}\right), 2.83$ $\left(\mathrm{dd}, 1 \mathrm{H}, J 4.5,16.3 \mathrm{~Hz}, \mathrm{COCH}_{\mathrm{a}} H_{\mathrm{b}}\right), 2.73(\mathrm{dd}, 1 \mathrm{H}, J$ $\left.4.2, \quad 16.4 \mathrm{~Hz}, \quad \mathrm{COCH}_{\mathrm{a}} \mathrm{H}_{\mathrm{b}}\right) ;{ }^{13} \mathrm{C} \quad \mathrm{NMR} \quad(100 \mathrm{MHz}$, $\left.\mathrm{CDCl}_{3}\right): \delta 168.7(\mathrm{C}=\mathrm{O}), 158.3,132.9,128.9(2 \mathrm{C})$, 128.0, 117.8, 113.2 (2C), 98.8, 79.1, 77.8, 71.6, 56.0 $\left(\mathrm{PhOCH}_{3}\right), 54.5\left(\mathrm{OCH}_{3}\right), 32.4\left(\mathrm{COCH}_{2}\right)$. Anal. Calcd for $\mathrm{C}_{16} \mathrm{H}_{20} \mathrm{O}_{5}$ : $\mathrm{C}, 65.74 ; \mathrm{H}, 6.90$. Found: $\mathrm{C}, 65.35 ; \mathrm{H}$, 6.82 .

\subsection{Methyl $(3 R, 4 R, 5 R)$-5-hydroxy-3-methoxy-4-(p- methoxybenzyloxy)-hept-6-enoate (17)}

To a soln of $7(600 \mathrm{mg}, 2.0 \mathrm{mmol})$ in anhyd $\mathrm{MeOH}$ $(15 \mathrm{~mL})$ was added $\mathrm{M} \mathrm{NaOMe}$ in $\mathrm{MeOH}$ until $\mathrm{pH} 9$ 
10. The reaction mixture was stirred at $\mathrm{rt}$ for $3 \mathrm{~h}$, then neutralized with Amberlite IR-120 $\left(\mathrm{H}^{+}\right)$, and filtered. The filtrate was concentrated to dryness under diminished pressure. Purification of the residue by column chromatography (4:1 petroleum ether-EtOAc) gave $\mathbf{1 7}$ (550 mg, 83\%) as a colorless syrup: $[\alpha]_{\mathrm{D}}^{25}-20(c 0.5$, $\left.\mathrm{CHCl}_{3}\right) ;{ }^{1} \mathrm{H}$ NMR $\left(\mathrm{CDCl}_{3}\right): \delta 7.26(\mathrm{~d}, 2 \mathrm{H}, J 8.6 \mathrm{~Hz}$, $\mathrm{Ph}), 6.88(\mathrm{~d}, 2 \mathrm{H}, J 8.6 \mathrm{~Hz}, \mathrm{Ph}), 6.00-5.90(\mathrm{~m}, 1 \mathrm{H}$, $\left.\mathrm{CH}=\mathrm{CH}_{2}\right), 5.44\left(\mathrm{~d}, 1 \mathrm{H}, J 17.2 \mathrm{~Hz}, \mathrm{CH}=\mathrm{CH}_{\mathrm{a}} \mathrm{H}_{\mathrm{b}}\right), 5.25$ $\left(\mathrm{d}, 1 \mathrm{H}, J 10.5 \mathrm{~Hz}, \mathrm{CH}=\mathrm{CH}_{\mathrm{a}} H_{\mathrm{b}}\right), 4.64,4.50(2 \mathrm{~d}, 2 \mathrm{H}, J$ $\left.11.3 \mathrm{~Hz}, \mathrm{PhCH}_{2}\right), 4.39(\mathrm{t}, 1 \mathrm{H}, J 5.3 \mathrm{~Hz}, \mathrm{CHOPMB})$, 4.02-3.98 $\left(\mathrm{m}, \quad 1 \mathrm{H}, \quad \mathrm{OCHCH}=\mathrm{CH}_{2}\right), 3.81 \quad(\mathrm{~s}, \quad 3 \mathrm{H}$, $\left.\mathrm{PhOCH}_{3}\right), 3.65$ (s, $\left.3 \mathrm{H}, \mathrm{CH}_{3} \mathrm{OOC}\right), 3.45(\mathrm{dd}, 1 \mathrm{H}, J 3.4$, $5.9 \mathrm{~Hz}, \mathrm{CHOMe}$ ), 3.33 (br s, $1 \mathrm{H}, \mathrm{OH}), 3.38$ (s, 3H, $\left.\mathrm{OCH}_{3}\right), 2.69\left(\mathrm{dd}, 1 \mathrm{H}, J 5.9,16.1 \mathrm{~Hz}, \mathrm{COCH}_{\mathrm{a}} H_{\mathrm{b}}\right), 2.61$ $\left(\mathrm{dd}, 1 \mathrm{H}, \quad J 7.0,16.1 \mathrm{~Hz}, \quad \mathrm{COCH}_{\mathrm{a}} \mathrm{H}_{\mathrm{b}}\right) ;{ }^{13} \mathrm{C}$ NMR $\left(100 \mathrm{MHz}, \mathrm{CDCl}_{3}\right): \delta 172.1(\mathrm{O}=\mathrm{COMe}), 159.4,137.9$, 129.8 (2C), 129.6, 116.1, 113.9 (2C), 78.8, 78.4, 72.3, 72.0, $58.0\left(\mathrm{COOCH}_{3}\right), 55.3\left(\mathrm{PhOCH}_{3}\right), 51.7\left(\mathrm{OCH}_{3}\right)$, $34.7\left(\mathrm{OOCCH}_{2}\right)$. Anal. Calcd for $\mathrm{C}_{17} \mathrm{H}_{24} \mathrm{O}_{6}$ : C, 62.95; $\mathrm{H}, 7.46$. Found: C, 62.67; H, 7.60.

\subsection{Methyl $(3 R, 4 R, 5 S)-5$-hydroxy-3-methoxy-4-(p- methoxybenzyloxy)-hept-6-enoate (18)}

To a soln of $17(520 \mathrm{mg}, 1.6 \mathrm{mmol})$ in dry THF (15 mL) was added $\mathrm{Ph}_{3} \mathrm{P}(839 \mathrm{mg}, 3.2 \mathrm{mmol})$, benzoic acid (391 $\mathrm{mg}, 3.2 \mathrm{mmol}$ ), and diethylazodicarboxylate (DEAD, $0.50 \mathrm{~mL}, 3.2 \mathrm{mmol}$ ) at $0{ }^{\circ} \mathrm{C}$. The resulting mixture was stirred at $\mathrm{rt}$ for $2 \mathrm{~h}$. After removing the solvent under diminished pressure, the residue was dissolved in EtOAc $(100 \mathrm{~mL})$ and washed with $\mathrm{N}$ aq $\mathrm{HCl}$, satd aq $\mathrm{NaHCO}_{3}$, and aq NaCl. After drying over anhyd $\mathrm{Na}_{2} \mathrm{SO}_{4}$ and concentration under diminished pressure, the residue was dissolved in anhyd $\mathrm{MeOH}$ $(15 \mathrm{~mL})$, and $\mathrm{M} \mathrm{NaOMe}$ in $\mathrm{MeOH}$ was added until $\mathrm{pH} 9-10$. The reaction mixture was stirred at $\mathrm{rt}$ for $3 \mathrm{~h}$ under these conditions, neutralized with Amberlite IR-120 $\left(\mathrm{H}^{+}\right)$, filtered, and concentrated. The residue was purified by silica gel column chromatography (4:1 petroleum ether-EtOAc) to yield 18 (421 mg, $81 \%)$ as a colorless syrup: $[\alpha]_{\mathrm{D}}^{25}-14\left(c 0.6, \mathrm{CHCl}_{3}\right)$; ${ }^{1} \mathrm{H}$ NMR $\left(\mathrm{CDCl}_{3}\right): \delta 7.25(\mathrm{~d}, 2 \mathrm{H}, J 8.4 \mathrm{~Hz}, \mathrm{Ph}), 6.89$ $(\mathrm{d}, 2 \mathrm{H}, J 8.4 \mathrm{~Hz}, \mathrm{Ph}), 6.05-5.96\left(\mathrm{~m}, 1 \mathrm{H}, \mathrm{CH}=\mathrm{CH}_{2}\right)$, $5.46\left(\mathrm{~d}, 1 \mathrm{H}, J 17.0 \mathrm{~Hz}, \mathrm{CH}=\mathrm{CH}_{\mathrm{a}} \mathrm{H}_{\mathrm{b}}\right), 5.28(\mathrm{~d}, 1 \mathrm{H}, J$ $\left.10.5 \mathrm{~Hz}, \mathrm{CH}=\mathrm{CH}_{\mathrm{a}} H_{\mathrm{b}}\right), 4.66,4.51(2 \mathrm{~d}, 2 \mathrm{H}, J 11.0 \mathrm{~Hz}$, $\left.\mathrm{PhCH}_{2}\right), 4.44-4.40(\mathrm{~m}, 1 \mathrm{H}, \mathrm{CHOPMB}), 4.02(\mathrm{t}, 1 \mathrm{H}, J$ $\left.6.9 \mathrm{~Hz}, \mathrm{OCHCH}=\mathrm{CH}_{2}\right), 3.80\left(\mathrm{~s}, 3 \mathrm{H}, \mathrm{PhOCH}_{3}\right), 3.68$ (s, 3H, $\left.\mathrm{CH}_{3} \mathrm{OOC}\right), 3.48(\mathrm{dd}, 1 \mathrm{H}, J 3.6,6.5 \mathrm{~Hz}$, $\mathrm{CHOMe}), 3.40\left(\mathrm{~s}, 3 \mathrm{H}, \mathrm{OCH}_{3}\right), 2.70-2.55(\mathrm{~m}, 2 \mathrm{H}$, $\left.\mathrm{COCH}_{2}\right) ;{ }^{13} \mathrm{C} \quad \mathrm{NMR} \quad\left(100 \mathrm{MHz}, \mathrm{CDCl}_{3}\right): \delta 172.0$ $(\mathrm{O}=\mathrm{COMe}), 159.5,137.8,129.8$ (2C), 129.5, 116.2, 113.8 (2C), 78.8, 78.3, 72.3, 71.9, $58.1\left(\mathrm{COOCH}_{3}\right)$, $55.2\left(\mathrm{PhOCH}_{3}\right), 51.8\left(\mathrm{OCH}_{3}\right), 34.6\left(\mathrm{OOCCH}_{2}\right)$. Anal. Calcd for $\mathrm{C}_{17} \mathrm{H}_{24} \mathrm{O}_{6}$ : C, 62.95; H, 7.46. Found: C, $62.70 ; \mathrm{H}, 7.35$.

\subsection{Methyl $(3 R, 4 S, 5 S)$-5-benzyloxy-3-methoxy-4-(p- methoxybenzyloxy)-hept-6-enoate (19)}

To a soln of 18 (250 mg, $0.77 \mathrm{mmol})$ and benzyl 2,2,2trichloroacetimidate $(380 \mathrm{mg}, 1.5 \mathrm{mmol})$ in dry $\mathrm{CH}_{2} \mathrm{Cl}_{2}$ $(15 \mathrm{~mL})$ at $-25^{\circ} \mathrm{C}$ was added TMSOTf $(3.0 \mu \mathrm{L}$, $0.008 \mathrm{mmol}$ ) under $\mathrm{N}_{2}$ atmosphere. The reaction was monitored by TLC until all the starting material was consumed, then quenched with $\mathrm{Et}_{3} \mathrm{~N}$ and concentrated to dryness. The residue was subjected to silica gel column chromatography (petroleum ether-EtOAc, 6:1) to give $19(240 \mathrm{mg}, 75 \%)$ as a colorless syrup: $[\alpha]_{\mathrm{D}}^{25}-16(c$ $\left.0.8, \mathrm{CHCl}_{3}\right) ;{ }^{1} \mathrm{H}$ NMR $\left(\mathrm{CDCl}_{3}\right): \delta 7.34-7.23(\mathrm{~m}, 7 \mathrm{H}$, $\mathrm{Ph}), 6.84(\mathrm{~d}, 2 \mathrm{H}, J 8.4 \mathrm{~Hz}, \mathrm{Ph}), 5.99-5.91(\mathrm{~m}, 1 \mathrm{H}$, $\left.\mathrm{CH}=\mathrm{CH}_{2}\right), 5.41\left(\mathrm{~d}, 1 \mathrm{H}, J 17.1 \mathrm{~Hz}, \mathrm{CH}=\mathrm{CH}_{\mathrm{a}} H_{\mathrm{b}}\right), 5.40$ $\left(\mathrm{d}, 1 \mathrm{H}, J 10.6 \mathrm{~Hz}, \mathrm{CH}=\mathrm{CH}_{\mathrm{a}} \mathrm{H}_{\mathrm{b}}\right), 4.65,4.62(2 \mathrm{~d}, 2 \mathrm{H}, J$ $\left.11.4 \mathrm{~Hz}, \mathrm{PhCH}_{2}\right), 4.46,4.34\left(2 \mathrm{~d}, 2 \mathrm{H}, J 11.3 \mathrm{~Hz}, \mathrm{PhCH}_{2}\right.$ ), $4.05(\mathrm{t}, 1 \mathrm{H}, J 6.9 \mathrm{~Hz}, \mathrm{CHOPMB}), 3.96-3.92(\mathrm{~m}, 1 \mathrm{H}$, $\left.\mathrm{OCHCH}=\mathrm{CH}_{2}\right), 3.79\left(\mathrm{~s}, 3 \mathrm{H}, \mathrm{PhOCH}_{3}\right), 3.64(\mathrm{~s}, 3 \mathrm{H}$, $\mathrm{CH}_{3} \mathrm{OOC}$ ), 3.57 (dd, $1 \mathrm{H}, J 3.5,6.3 \mathrm{~Hz}, \mathrm{CHOMe}$ ), 3.31 (s, $\left.3 \mathrm{H}, \mathrm{OCH}_{3}\right), 2.58-2.41\left(\mathrm{~m}, 2 \mathrm{H}, \mathrm{COCH}_{2}\right) ;{ }^{13} \mathrm{C} \mathrm{NMR}$ $\left(100 \mathrm{MHz}, \mathrm{CDCl}_{3}\right): \delta 172.6(\mathrm{O}=\mathrm{COMe}), 158.9,138.6$, $135.5,130.4,129.1$ (2C), $127.6(2 \mathrm{C}), 127.0$ (2C), 126.8, $119.5,113.5$ (2C), 81.6, 80.1, 72.6, 70.0, 69.3, 58.1

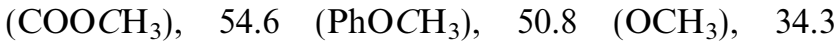
$\left(\mathrm{OOCCH}_{2}\right)$. Anal. Calcd for $\mathrm{C}_{24} \mathrm{H}_{30} \mathrm{O}_{6}: \mathrm{C}, 69.54 ; \mathrm{H}$, 7.30. Found: C, 69.91; H, 7.21.

\section{9. (S)-Hept-6-en-2-yl $(3 R, 4 S, 5 S)$-5-benzyloxy-3-meth- oxy-4-(p-methoxybenzyloxy)-hept-6-enoate (20)}

Methyl ester 19 (210 mg, $0.51 \mathrm{mmol})$ was dissolved in 4:1 THF-water $(15 \mathrm{~mL})$ and the soln was cooled in an ice-water bath. To this was added $\mathrm{LiOH} \cdot \mathrm{H}_{2} \mathrm{O}(84 \mathrm{mg}$, $2 \mathrm{mmol}$ ), and the resulting mixture was stirred at room temperature for $12 \mathrm{~h}$, neutralized with $1 \mathrm{~N} \mathrm{HCl}$, and then extracted with EtOAc. The combined organic phases were dried with anhyd $\mathrm{Na}_{2} \mathrm{SO}_{4}$ and concentrated to afford acid $\mathbf{6}$. Without further purification, the crude acid was dissolved in anhyd THF $(15 \mathrm{~mL})$ and treated with $\mathrm{Et}_{3} \mathrm{~N}(82 \mu \mathrm{L}, 0.6 \mathrm{mmol})$ and 2,4,6-trichlorobenzoyl chloride ( $78 \mu \mathrm{L}, 0.5 \mathrm{mmol})$ successively. After stirring at $\mathrm{rt}$ for $1 \mathrm{~h}$, a soln of $(S)$-6-hepten-2-ol $(68 \mathrm{mg}, 0.6 \mathrm{mmol})$ and DMAP $(73 \mathrm{mg}, 0.6 \mathrm{mmol})$ in THF $(5 \mathrm{~mL})$ was added. The reaction mixture was stirred at $\mathrm{rt}$ for another $18 \mathrm{~h}$, then diluted with aq $\mathrm{NH}_{4} \mathrm{Cl}$. The aq soln was extracted with EtOAc $(2 \times 25 \mathrm{~mL})$. The combined organic extracts was dried and evaporated. Purification of the residue by column chromatography (6:1 petroleum ether-EtOAc) gave 20 (196 mg, 78\% in two steps) as a colorless syrup: $[\alpha]_{\mathrm{D}}^{25}-22\left(c 0.5, \mathrm{CHCl}_{3}\right) ;{ }^{1} \mathrm{H}$ NMR $\left(\mathrm{CDCl}_{3}\right): \delta$ 7.34-7.24 (m, 7H, Ph), $6.84(\mathrm{~d}, 2 \mathrm{H}, J$ $8.0 \mathrm{~Hz}, \mathrm{Ph}), 5.97-5.92\left(\mathrm{~m}, 1 \mathrm{H}, \mathrm{CH}=\mathrm{CH}_{2}\right), 5.78-5.74$ $\left(\mathrm{m}, 1 \mathrm{H}, \mathrm{CH}=\mathrm{CH}_{2}\right), 5.42\left(\mathrm{~d}, 1 \mathrm{H}, J 9.0 \mathrm{~Hz} \mathrm{CH}=\mathrm{CH}_{\mathrm{a}} \mathrm{H}_{\mathrm{b}}\right)$, $5.38\left(\mathrm{~d}, 1 \mathrm{H}, J 18.0 \mathrm{~Hz}, \mathrm{CH}=\mathrm{CH}_{\mathrm{a}} H_{\mathrm{b}}\right), 5.00(\mathrm{~d}, 1 \mathrm{H}, J$ $\left.17.4 \mathrm{~Hz}, \mathrm{CH}=\mathrm{CH}_{\mathrm{a}} H_{\mathrm{b}}\right), 4.98-4.90\left(\mathrm{~m}, 2 \mathrm{H}, \mathrm{CH}=\mathrm{CH}_{\mathrm{a}} \mathrm{H}_{\mathrm{b}}\right.$, 
CHOOC), 4.65, $4.48\left(2 \mathrm{~d}, 2 \mathrm{H}, J 10.5 \mathrm{~Hz}, \mathrm{PhCH}_{2}\right), 4.62$, $4.36\left(2 \mathrm{~d}, 2 \mathrm{H}, J 11.2 \mathrm{~Hz}, \mathrm{PhCH}_{2}\right), 4.02(\mathrm{t}, 1 \mathrm{H}, J 6.8 \mathrm{~Hz}$, CHOPMB $), 3.97-3.95\left(\mathrm{~m}, 1 \mathrm{H}\right.$, OCHCH$\left.=\mathrm{CH}_{2}\right), 3.80$ $\left(\mathrm{s}, 3 \mathrm{H}, \mathrm{PhOCH}_{3}\right), 3.57-3.55(\mathrm{~m}, 1 \mathrm{H}, \mathrm{CHOMe}), 3.32(\mathrm{~s}$, $\left.3 \mathrm{H}, \mathrm{OCH}_{3}\right), 2.60-2.47\left(\mathrm{~m}, 2 \mathrm{H}, \mathrm{COCH}_{2}\right), 2.04(\mathrm{dd}, 2 \mathrm{H}$, $J$ 6.7, $\left.13.3 \mathrm{~Hz}, \quad \mathrm{CH}_{2} \mathrm{CH}=\mathrm{CH}_{2}\right), 1.55-1.41(\mathrm{~m}, 4 \mathrm{H}$, $\left.\mathrm{CH}_{2} \mathrm{CH}_{2}\right), 1.21\left(\mathrm{~d}, 3 \mathrm{H}, J 6.0 \mathrm{~Hz}, \mathrm{CH}_{2} \mathrm{CH}_{3}\right) ;{ }^{13} \mathrm{C} \mathrm{NMR}$ $\left(100 \mathrm{MHz}, \mathrm{CDCl}_{3}\right): \delta 171.5(\mathrm{O}=\mathrm{COCH}), 159.2,138.4$ (2C), 136.2, 133.3, 130.4 (2C), 129.8, 128.2 (2C), 127.7, 127.4 (2C), 119.4, 114.7, 113.6 (2C), 81.8, 80.1, 77.5, 74.0, 70.9, 58.6 $\left(\mathrm{OCH}_{3}\right), 55.2\left(\mathrm{PhOCH}_{3}\right), 36.5,35.3$, 33.4, 24.5, 19.9. Anal. Calcd for $\mathrm{C}_{30} \mathrm{H}_{40} \mathrm{O}_{6}$ : C, 72.55; $\mathrm{H}, 8.12$. Found: C, 72.81; H, 8.01.

\subsection{0. (S)-Hept-6-en-2-yl $(3 R, 4 S, 5 S)$-5-benzyloxy-4- hydroxy-3-methoxy-hept-6-enoate (21)}

A soln of ester $20(170 \mathrm{mg}, 0.33 \mathrm{mmol})$ in $\mathrm{CH}_{2} \mathrm{Cl}_{2}$ $(10 \mathrm{~mL})$ at $0{ }^{\circ} \mathrm{C}$ was treated with water $(1 \mathrm{~mL})$ and DDQ (150 mg, $0.66 \mathrm{mmol}$ ), and then stirred at $\mathrm{rt}$ for $2 \mathrm{~h}$. Satd aq $\mathrm{NaHCO}_{3}$ was added, and the aq layer was extracted with $\mathrm{CH}_{2} \mathrm{Cl}_{2}(2 \times 20 \mathrm{~mL})$. The combined organic extracts were dried over anhyd $\mathrm{Na}_{2} \mathrm{SO}_{4}$ and concentrated to dryness. Column chromatography of the residue (4:1 petroleum ether-EtOAc) gave $\mathbf{2 1}$ $(120 \mathrm{mg}, 93 \%)$ as a colorless syrup: $[\alpha]_{\mathrm{D}}^{25}-25(c 0.5$, $\left.\mathrm{CHCl}_{3}\right),{ }^{1} \mathrm{H}$ NMR $\left(\mathrm{CDCl}_{3}\right): \delta 7.34-7.26(\mathrm{~m}, 5 \mathrm{H}, \mathrm{Ph})$, 5.86-5.82 $\left(\mathrm{m}, 1 \mathrm{H}, \mathrm{C} H=\mathrm{CH}_{2}\right), \quad 5.77-5.73(\mathrm{~m}, 1 \mathrm{H}$, $\left.\mathrm{CH}=\mathrm{CH}_{2}\right), 5.43\left(\mathrm{~d}, 1 \mathrm{H}, J 10.2 \mathrm{~Hz}, \mathrm{CH}=\mathrm{CH}_{\mathrm{a}} \mathrm{H}_{\mathrm{b}}\right), 5.38$ $\left(\mathrm{d}, 1 \mathrm{H}, J 17.3 \mathrm{~Hz}, \quad \mathrm{CH}=\mathrm{CH}_{\mathrm{a}} H_{\mathrm{b}}\right), 5.00(\mathrm{~d}, 1 \mathrm{H}, J$ $\left.17.3 \mathrm{~Hz}, \mathrm{CH}=\mathrm{CH}_{\mathrm{a}} H_{\mathrm{b}}\right), 4.96-4.91\left(\mathrm{~m}, 2 \mathrm{H}, \mathrm{CH}=\mathrm{CH}_{\mathrm{a}} \mathrm{H}_{\mathrm{b}}\right.$, CHOOC), 4.63, $4.36\left(2 \mathrm{~d}, 2 \mathrm{H}, J 11.6 \mathrm{~Hz}, \mathrm{PhCH}_{2}\right)$, 3.99 (br s, $1 \mathrm{H}, \mathrm{CHOH}), 3.81(\mathrm{t}, 1 \mathrm{H}, J 7.4 \mathrm{~Hz}$, $\left.\mathrm{OCHCH}=\mathrm{CH}_{2}\right), 3.49(\mathrm{~d}, 1 \mathrm{H}, J 6.5 \mathrm{~Hz}, \mathrm{CHOMe}), 3.34$ (s, $\left.3 \mathrm{H}, \mathrm{OCH}_{3}\right), 2.67-2.55\left(\mathrm{~m}, 2 \mathrm{H}, \mathrm{COCH}_{2}\right), 2.04(\mathrm{dd}$, $\left.2 \mathrm{H}, J 6.8,13.6 \mathrm{~Hz}, \mathrm{CH}_{2} \mathrm{CH}=\mathrm{CH}_{2}\right), 1.55-1.36(\mathrm{~m}, 4 \mathrm{H}$, $\left.\mathrm{CH}_{2} \mathrm{CH}_{2}\right), 1.20\left(\mathrm{~d}, 3 \mathrm{H}, J 6.1 \mathrm{~Hz}, \mathrm{CH}_{2} \mathrm{CH}_{3}\right) ;{ }^{13} \mathrm{C} \mathrm{NMR}$ $\left(100 \mathrm{MHz}, \mathrm{CDCl}_{3}\right): \delta 171.3(\mathrm{O}=\mathrm{COCH}), 138.4,138.1$, 136.1, 128.3 (2C), 128.0 (2C), 127.6, 120.2, 114.7, 80.5, 76.1, 74.7, 71.1, 70.2, $58.7\left(\mathrm{OCH}_{3}\right), 37.0,35.3,33.4$, 24.6, 19.9. Anal. Calcd for $\mathrm{C}_{22} \mathrm{H}_{32} \mathrm{O}_{5}: \mathrm{C}, 70.18 ; \mathrm{H}$, 8.57. Found: C, 69.89; H, 8.48.

\subsection{1. (S)-Hept-6-en-2-yl $(3 R, 5 S)$-5-benzyloxy-3-meth- oxy-4-oxo-hept-6-enoate (4)}

To a soln of 21 (970 mg, $2.6 \mathrm{mmol})$ in dry $\mathrm{CH}_{2} \mathrm{Cl}_{2}$ $(150 \mathrm{~mL})$, Dess-Martin periodinane $(1.65 \mathrm{~g}, 3.9 \mathrm{mmol})$ was added and the reaction mixture was stirred for $3 \mathrm{~h}$ at rt. After extraction with satd aq $\mathrm{NaHCO}_{3}$, the organic layer was separated, dried, and concentrated. The residue was subjected to column chromatography on silica gel (6:1 petroleum ether-EtOAc) to furnish compound 4 $(802 \mathrm{mg}, 83 \%)$ as a colorless syrup: $[\alpha]_{\mathrm{D}}^{25}-30(c 0.5$, $\left.\mathrm{CHCl}_{3}\right) ;{ }^{1} \mathrm{H}$ NMR $\left(\mathrm{CDCl}_{3}\right): \delta 7.40-7.30(\mathrm{~m}, 5 \mathrm{H}, \mathrm{Ph})$, 5.90-5.86 $\left(\mathrm{m}, 1 \mathrm{H}, \mathrm{CH}=\mathrm{CH}_{2}\right), 5.77-5.74(\mathrm{~m}, 1 \mathrm{H}$,
$\left.\mathrm{CH}=\mathrm{CH}_{2}\right), 5.50\left(\mathrm{~d}, 1 \mathrm{H}, J 17.3 \mathrm{~Hz}, \mathrm{CH}=\mathrm{CH}_{\mathrm{a}} H_{\mathrm{b}}\right), 5.42$ $\left(\mathrm{d}, \quad 1 \mathrm{H}, J 10.3 \mathrm{~Hz}, \quad \mathrm{CH}=\mathrm{CH}_{\mathrm{a}} \mathrm{H}_{\mathrm{b}}\right), \quad 5.00 \quad(\mathrm{~d}, 1 \mathrm{H}, J$ $\left.17.2 \mathrm{~Hz}, \mathrm{CH}=\mathrm{CH}_{\mathrm{a}} H_{\mathrm{b}}\right), 4.97-4.92\left(\mathrm{~m}, 2 \mathrm{H}, \mathrm{CH}=\mathrm{CH}_{\mathrm{a}} \mathrm{H}_{\mathrm{b}}\right.$, $\mathrm{C} H \mathrm{OOC}), 4.62\left(\mathrm{~d}, 1 \mathrm{H}, J 6.7 \mathrm{~Hz}, \mathrm{OC} H \mathrm{CH}=\mathrm{CH}_{2}\right), 4.67$, $4.54\left(2 \mathrm{~d}, 2 \mathrm{H}, J 11.9 \mathrm{~Hz}, \mathrm{PhCH}_{2}\right), 4.48(\mathrm{dd}, 1 \mathrm{H}, J 3.6$, $8.2 \mathrm{~Hz}, \mathrm{CHOMe}$ ), 3.34 (s, $\left.3 \mathrm{H}, \mathrm{OCH}_{3}\right), 2.77(\mathrm{dd}, 1 \mathrm{H}, J$ $\left.3.7,16.1 \mathrm{~Hz}, \mathrm{COCH}_{\mathrm{a}} H_{\mathrm{b}}\right), 2.52(\mathrm{dd}, 1 \mathrm{H}, J 8.4,16.1 \mathrm{~Hz}$, $\left.\mathrm{COCH}_{\mathrm{a}} \mathrm{H}_{\mathrm{b}}\right), \quad 2.04(\mathrm{dd}, 2 \mathrm{H}, \quad J \quad 7.0, \quad 13.8 \mathrm{~Hz}$, $\left.\mathrm{CH}_{2} \mathrm{CH}=\mathrm{CH}_{2}\right), 1.53-1.43\left(\mathrm{~m}, 4 \mathrm{H}, \mathrm{CH}_{2} \mathrm{CH}_{2}\right), 1.20(\mathrm{~d}$, $\left.3 \mathrm{H}, J 6.1 \mathrm{~Hz}, \mathrm{CH}_{2} \mathrm{CH}_{3}\right) ;{ }^{13} \mathrm{C}$ NMR $\left(100 \mathrm{MHz}, \mathrm{CDCl}_{3}\right)$ : $\delta 206.6(\mathrm{C}=\mathrm{O}), 169.9(\mathrm{O}=\mathrm{COCH}), 138.4,137.2,132.3$, 128.5 (2C), 128.0, 127.9 (2C), 120.1, 114.7, 83.1, 80.5, 71.6, 71.4, $58.7\left(\mathrm{OCH}_{3}\right), 37.1,35.3,33.4,24.5,19.9$. Anal. Calcd for $\mathrm{C}_{22} \mathrm{H}_{30} \mathrm{O}_{5}: \mathrm{C}, 70.56 ; \mathrm{H}, 8.07$. Found: C, 70.91; H, 7.88.

\subsection{Lactone 3}

Diolefin 4 (550 mg, $1.4 \mathrm{mmol})$, dissolved in dry degassed $\mathrm{CH}_{2} \mathrm{Cl}_{2}(100 \mathrm{~mL})$, was added dropwise within $2 \mathrm{~h}$ to a refluxing soln of ruthenium catalyst ${ }^{17}(360 \mathrm{mg}$, $0.4 \mathrm{mmol})$ in dry degassed $\mathrm{CH}_{2} \mathrm{Cl}_{2}(2 \mathrm{~L})$. The mixture was heated at reflux until complete consumption of the starting material (20-24 h, TLC monitoring). After solvent removal under diminished pressure, the residue was purified by column chromatography $(8: 1$ petroleum ether-EtOAc) yielding first (Z)-3 (120 mg, 23.5\%) and then $(E)-3(250 \mathrm{mg}, 46.5 \%)$ as colorless syrups: for the $Z$-isomer, $[\alpha]_{\mathrm{D}}^{25}-28\left(c 0.5, \mathrm{CHCl}_{3}\right) ;{ }^{1} \mathrm{H} \mathrm{NMR}\left(\mathrm{CDCl}_{3}\right)$ : $\delta \quad 7.35-7.26(\mathrm{~m}, \quad 5 \mathrm{H}, \quad \mathrm{Ph}), \quad 5.83-5.79(\mathrm{~m}, \quad 1 \mathrm{H}$, $\left.\mathrm{CH}_{2} \mathrm{CH}=\mathrm{CH}\right), 5.62\left(\mathrm{t}, 1 \mathrm{H}, J 9.5 \mathrm{~Hz}, \mathrm{CH}_{2} \mathrm{CH}=\mathrm{CH}\right)$, 4.89 (d, $1 \mathrm{H}, J 8.7 \mathrm{~Hz}, \mathrm{CHOOC}), 4.83(\mathrm{dd}, 1 \mathrm{H}, J 6.3$, $11.8 \mathrm{~Hz}, \mathrm{CHOBn}), 4.62(\mathrm{~d}, 1 \mathrm{H}, J 11.7 \mathrm{~Hz}, \mathrm{PhCH})$, $4.52(\mathrm{~d}, 1 \mathrm{H}, J 11.5 \mathrm{~Hz}, \mathrm{PhCH}), 3.40\left(\mathrm{~s}, 3 \mathrm{H}, \mathrm{OCH}_{3}\right)$, $4.34(\mathrm{dd}, 1 \mathrm{H}, J$ 2.7, $9.4 \mathrm{~Hz}, \mathrm{CHOMe}), 2.91(\mathrm{dd}, 1 \mathrm{H}, J$ 2.6, $\left.14.8 \mathrm{~Hz}, \quad \mathrm{OOCCH}_{\mathrm{a}} H_{\mathrm{b}}\right), 2.70(\mathrm{dd}, 1 \mathrm{H}, J$ 9.3, $\left.14.8 \mathrm{~Hz}, \mathrm{OOCCH}_{\mathrm{a}} \mathrm{H}_{\mathrm{b}}\right), 2.08(\mathrm{dd}, 2 \mathrm{H}, J 9.7,16.9 \mathrm{~Hz}$, $\left.\mathrm{CH}_{2} \mathrm{CH}=\mathrm{CH}\right), 1.53-1.44\left(\mathrm{~m}, 4 \mathrm{H}, \mathrm{CH}_{2} \mathrm{CH}_{2}\right), 1.20(\mathrm{~d}$, $\left.3 \mathrm{H}, J 6.1 \mathrm{~Hz}, \mathrm{CH}_{2} \mathrm{CH}_{3}\right) ;{ }^{13} \mathrm{C} \mathrm{NMR}\left(100 \mathrm{MHz}, \mathrm{CDCl}_{3}\right)$ : $\delta 205.6(\mathrm{C}=\mathrm{O}), 169.1(\mathrm{O}=\mathrm{COCH}), 137.3,128.3(2 \mathrm{C})$, 128.1, 128.0 (2C), 127.8, 125.3, 79.5, 76.1, 72.3, 71.1, $58.2\left(\mathrm{OCH}_{3}\right), 37.1,32.7,27.0,22.7,20.6$; for the $E$-isomer, $[\alpha]_{\mathrm{D}}^{25}-26\left(c \quad 0.5, \mathrm{CHCl}_{3}\right),{ }^{1} \mathrm{H}$ NMR $\left(\mathrm{CDCl}_{3}\right): \delta$ 7.39-7.31 (m, 5H, Ph), 5.91-5.87 (m, 1H, $\left.\mathrm{CH}_{2} \mathrm{CH}=\mathrm{CH}\right)$, $5.42\left(\mathrm{dd}, 1 \mathrm{H}, J 4.7,15.7 \mathrm{~Hz}, \mathrm{CH}_{2} \mathrm{CH}=\mathrm{CH}\right), 4.87(\mathrm{t}, 1 \mathrm{H}$, $J 5.6 \mathrm{~Hz}, \mathrm{CHOOC}), 4.63(\mathrm{~d}, 1 \mathrm{H}, J 11.9 \mathrm{~Hz}, \mathrm{PhCH}), 4.61$ $(\mathrm{d}, 1 \mathrm{H}, J 7.0 \mathrm{~Hz}, \mathrm{CHOBn}), 4.54(\mathrm{~d}, 1 \mathrm{H}, J 11.8 \mathrm{~Hz}$, $\mathrm{PhCH}), 3.42\left(\mathrm{~s}, 3 \mathrm{H}, \mathrm{OCH}_{3}\right), 4.19(\mathrm{dd}, 1 \mathrm{H}, J 3.1$, $6.7 \mathrm{~Hz}, \mathrm{CHOMe}), 3.11(\mathrm{dd}, 1 \mathrm{H}, J 3.0,16.2 \mathrm{~Hz}$, OOC$\left.\mathrm{CH}_{\mathrm{a}} H_{\mathrm{b}}\right), 2.85\left(\mathrm{dd}, 1 \mathrm{H}, J 6.7,16.3 \mathrm{~Hz}, O O O C H_{\mathrm{a}} \mathrm{H}_{\mathrm{b}}\right)$, 2.19-2.17 (m, $\left.1 \mathrm{H}, \mathrm{CH}_{\mathrm{a}} H_{\mathrm{b}} \mathrm{CH}=\mathrm{CH}\right), 1.92-1.89(\mathrm{~m}, 1 \mathrm{H}$, $\left.\mathrm{CH}_{\mathrm{a}} \mathrm{H}_{\mathrm{b}} \mathrm{CH}=\mathrm{CH}\right), 1.55-1.47\left(\mathrm{~m}, 4 \mathrm{H}, \mathrm{CH}_{2} \mathrm{CH}_{2}\right), 1.14$ $\left(\mathrm{d}, 3 \mathrm{H}, J 6.5 \mathrm{~Hz}, \mathrm{CH}_{2} \mathrm{CH}_{3}\right) ;{ }^{13} \mathrm{C}$ NMR $(100 \mathrm{MHz}$, $\left.\mathrm{CDCl}_{3}\right): \delta 204.3(\mathrm{C}=\mathrm{O}), 169.0(\mathrm{O}=\mathrm{COCH}), 137.5$, 133.7, 128.5 (2C), 127.9, 127.6 (2C), 124.4, 83.5, 79.6, 71.9, 71.2, $58.4\left(\mathrm{OCH}_{3}\right), 35.9,32.1,31.6,21.7,19.1$; 
$\mathrm{ESI}^{(+)} \mathrm{MS}: m / z 347[\mathrm{M}+\mathrm{H}]^{+}, 369[\mathrm{M}+\mathrm{Na}]^{+}$. Anal. Calcd for $\mathrm{C}_{20} \mathrm{H}_{26} \mathrm{O}_{5}$ ( $E, Z$ mixture): C, 69.34; $\mathrm{H}, 7.56$. Found: C, 69.05; H, 7.46.

\subsection{Sporiolide B (1)}

To a suspension of $\mathrm{Pd} / \mathrm{C}(5 \% \mathrm{Pd}, 100 \mathrm{mg})$ in $\mathrm{MeOH}$ $(50 \mathrm{~mL})$ was added a soln of $\mathbf{3}(E / Z$ mixture, $250 \mathrm{mg}$, $0.72 \mathrm{mmol})$ in $\mathrm{MeOH}(20 \mathrm{~mL})$. The mixture was allowed to stir at $\mathrm{rt}$ under $\mathrm{H}_{2}$ atmosphere. After $12 \mathrm{~h}$, the reaction mixture was filtered and concentrated under diminished pressure. The residue was subjected to column chromatography on silica gel $(5: 1$ petroleum etherEtOAc) to give sporiolide B (150 mg, $81 \%)$ as a colorless amorphous solid: $[\alpha]_{\mathrm{D}}^{25}-32(c 0.5, \mathrm{MeOH}) ;$ lit. $^{4}[\alpha]_{\mathrm{D}}^{25}-29$ $\left(c 0.5, \mathrm{CHCl}_{3}\right),[\alpha]_{\mathrm{D}}^{25}-33(c 0.5, \mathrm{MeOH}) ;$ lit. $^{3}[\alpha]_{\mathrm{D}}^{25}-33(c$ $0.3, \mathrm{MeOH}) ;{ }^{1} \mathrm{H}$ NMR $\left(\mathrm{CDCl}_{3}\right): \delta 4.81-4.83(\mathrm{~m}, 1 \mathrm{H}, \mathrm{H}-$ 11), 4.44 (d, $1 \mathrm{H}, J 8.7 \mathrm{~Hz}, \mathrm{H}-3), 4.30(\mathrm{~d}, 1 \mathrm{H}, J 5.7 \mathrm{~Hz}$, $\mathrm{H}-5), 3.53\left(\mathrm{~s}, 3 \mathrm{H}, \mathrm{OCH}_{3}\right), 3.43(\mathrm{t}, 1 \mathrm{H}, J 12.6 \mathrm{~Hz}, \mathrm{H}-$ 2b), $2.60(\mathrm{dd}, 1 \mathrm{H}, J 8.8,13.9 \mathrm{~Hz}, \mathrm{H}-2 \mathrm{a}), 2.04-2.06(\mathrm{~m}$, 1H, H-6b), 1.58-1.69 (m, 2H, H-10b, H-6a), 1.48-1.57 (m, 2H, H-7b, H-9b), 1.38-1.47 (m, 2H, H-8b, H-9a), 1.25-1.36 (m, 2H, H-8a, H-10a), 1.23 (d, 3H, J 6.4 Hz, $\mathrm{H}-12), 1.07-1.09$ (m, 1H, H-7a); ${ }^{13} \mathrm{C}$ NMR $(100 \mathrm{MHz}$, $\left.\mathrm{CDCl}_{3}\right): \delta 208.8(\mathrm{C}-4), 170.6(\mathrm{C}-1), 76.1$ (C-5), $74.4(\mathrm{C}-$ 3), $73.3(\mathrm{C}-11), 58.4\left(\mathrm{OCH}_{3}\right), 41.4(\mathrm{C}-2), 33.4(\mathrm{C}-10)$, 30.0 (C-6), 26.5 (C-8), 23.8 (C-9), 22.2 (C-7), 20.8 (C12); MALDI-FTMS: calcd for $\mathrm{C}_{13} \mathrm{H}_{22} \mathrm{O}_{5}: 258.1467$ $[\mathrm{M}]^{+}$; found: $281.1403[\mathrm{M}+\mathrm{Na}]^{+}$.

\section{Acknowledgments}

This work was supported by the National Basic Research Program of China (2003CB415001), and NNSF of China (Projects 20621703 and 20572128).

\section{Supplementary data}

Supplementary data associated with this article can be found, in the online version, at doi:10.1016/j.carres. 2007.04.013.

\section{References}

1. (a) Blunt, J. W.; Copp, B. R.; Munro, M. H. G.; Northcote, P. T.; Prinsep, M. R. Nat. Prod. Rep. 2004, 21, 1-49; (b) Faulkner, D. Nat. Prod. Rep. 2002, 19, 1-48; (c) Blunt, J. W.; Copp, B. R.; Munro, M. H. G.; Northcote, P. T.; Prinsep, M. R. Nat. Prod. Rep. 2005, 22, 15-61; (d) Blunt, J. W.; Copp, B. R.; Munro, M. H. G.; Northcote, P. T.; Prinsep, P. R. Nat. Prod. Rep. 2006, 23, 26-78; (e) Tsuda, M.; Mugishima, T.; Komatsu, K.; Sone, T.; Tanaka, M. J. Nat. Prod. 2003, 66, 412-415.

2. Gesner, S.; Cohen, N.; Iian, M.; Yarden, O.; Carmeli, S. J. Nat. Prod. 2005, 68, 1350-1353.

3. Shigemori, H.; Kasai, Y.; Komatsu, K.; Tsuda, M.; Mikami, Y.; Kobayashi, J. Mar. Drugs 2004, 2, 164-169.

4. Chen, Q.; Du, Y. Tetrahedron Lett. 2006, 47, 8489-8492.

5. Schell, P.; Orgueira, H. A.; Roehrig, S.; Seeberger, P. H. Tetrahedron Lett. 2001, 42, 3811-3814.

6. Riley, A. M.; Jenkins, D. J.; Marwood, R. D.; Potter, B. V. L. Carbohydr. Res. 2002, 337, 1067-1082.

7. Joniak, D.; Košíková, B.; Kosáková, L. Collect. Czech. Chem. Commun. 1978, 43, 769-773.

8. Johanssonm, R.; Samuelsson, B. J. Chem. Soc., Perkin Trans. 1 1984, 2371-2374.

9. Du, Y.; Chen, Q.; Linhardt, R. J. J. Org. Chem. 2006, 71, 8446-8451.

10. (a) Rollin, P.; Sinaÿ, P. Carbohydr. Res. 1981, 98, 139-142; (b) Roth, B. D.; Roark, W. H. Tetrahedron Lett. 1988, 29, 1255-1258; (c) Baba, T.; Huang, G.; Isobe, M. Tetrahedron 2003, 59, 6851-6872; (d) Piancatelli, G.; Scettri, A.; D'Auria, M. Tetrahedron Lett. 1977, 18, 3483-3484.

11. (a) Mitsunobu, O. Synthesis 1981, 1-28; (b) Ahn, C.; Correia, R.; DeShong, P. J. Org. Chem. 2002, 67, 17511753, and references cited therein.

12. Du, Y.; Liu, J.; Linhardt, R. J. J. Org. Chem. 2006, 71, 1251-1253.

13. Takahata, H.; Yotsui, Y.; Momose, T. Tetrahedron 1998, 54, 13505-13516.

14. Inanaga, J.; Hirata, K.; Saeki, H.; Katsuki, T.; Yamaguchi, M. Bull. Chem. Soc. Jpn. 1979, 52, 1989-1993.

15. (a) Oikawa, Y.; Tanaka, T.; Horita, K.; Yonemitsu, O. Tetrahedron Lett. 1984, 25, 5397-5400; (b) Tanaka, T.; Oikawa, Y.; Hamada, T.; Yonemitsu, O. Tetrahedron Lett. 1986, 27, 3651-3654.

16. Dess, P. B.; Martin, J. C. J. Org. Chem. 1983, 48, 41554156.

17. Grubbs, R. H.; Chang, S. Tetrahedron 1998, 54, 44134450 .

18. (a) Kuroda, I.; Musman, M.; Ohtani, I. I.; Ichiba, T.; Tanaka, J.; Cravalos, D. C.; Higa, T. J. Nat. Prod. 2002, 65, 1505-1506; (b) Ledroit, V.; Debitus, C.; Lavaud, C.; Massiot, G. Tetrahedron Lett. 2003, 44, 225-228. 